\title{
APORTACIONES DE LA ÉTICA DE LA ALTERIDAD DE E. LÉVINAS Y LA ÉTICA DEL CUIDADO DE C. GILLIGAN A LA INTERVENCIÓN EN TRABAJO SOCIAL
}

\author{
Contributions of E. Lévina's Ethics of Alterity and \\ C. Gilligan's Ethics of Care in Social Work Intervention \\ FRANCISCO IDARETA GOLDARACENA ${ }^{1}$ \\ MARÍA Jesús Úriz PEMÁN ${ }^{2}$
}

\section{Resumen}

$\mathrm{Al}$ igual que en otras profesiones, la dimensión deontológica del Trabajo Social es la parte de la ética profesional que ha alcanzado un desarrollo mayor. La expresión más clara y conocida del deontologismo son los principios éticos, que constituyen el fundamento sobre el que se asientan las intervenciones profesionales. En nuestra opinión, la aplicación de principios éticos universales racionales es necesaria, pero no suficiente. Por ello, a lo largo de este artículo nos proponemos ampliar la perspectiva principialista con dos aportaciones que creemos pueden ser de gran utilidad en las intervenciones profesionales: la ética de la alteridad de E. Lévinas y la ética del cuidado de C. Gilligan. Expondremos cómo la aplicación de ambas perspectivas éticas redundaría en una mayor humanización de las intervenciones sociales.

El artículo se estructura de la siguiente forma: tras una breve introducción al tema, en una primera parte se expondrá la ética de la alteridad de Lévinas, en una segunda la ética del cuidado de Gilligan, mientras que en una tercera se procederá a considerarlas en el contexto concreto del Trabajo Social. Finalmente, y de modo escueto, presentamos las conclusiones.

Palabras Clave: Ética del Trabajo Social - E. Lévinas - C. Gilligan - sensibilidad cuidado.

\begin{abstract}
As in other professions, the ethical dimension of social work is the part of professional ethics that has developed to a greater degree. The clearest expression of deontologism is ethical principles, which constitute the foundation on which professional interventions are based. In our opinion, the application of rational universal ethical principles is necessary but not sufficient. Therefore, throughout this article we propose to extend the perspective of these principles with two contributions that we believe may be useful in professional interventions: E. Lévina's ethics of alterity and C. Gilligan's ethics of care. We will show how the application of these two ethical perspectives would enhance the humanisation of social interventions.

The paper is structured as follows: after a short introduction, in the first part, the ethics of alterity of Levinas will be outlined. In the second part, the ethics of care of Gilligan will be explained, after that they will be considered in the specific context of Social Work. Finally, the conclusions will be briefly presented.
\end{abstract}

Key words: Social Work Ethics - E. Lévinas

- C. Gilligan - sensitivity - care.

\begin{tabular}{llll}
\hline Recibido: 15/12/2010 & Revisado: 03/11/2011 & Aceptado: 14/12/2011 & Publicado: 02/11/2012 \\
\hline
\end{tabular}

1. Francisco Idareta Goldaracena. Universidad Pública de Navarra. Dpto. de Trabajo Social.

2. María Jesús Úriz Pemán. Universidad Pública de Navarra. Dpto. de Trabajo Social. Campus de Arrosadía s/n. 31006-Pamplona. E-mail: ivan@unavarra.es 


\section{Introducción}

La preponderancia del principialismo ético en el Trabajo Social es una realidad innegable. Muchas de las formulaciones de principios éticos (autonomía, bienestar, justicia, etc.) tienen su origen filosófico en la ética kantiana, así como en el utilitarismo consecuencialista de J.S. Mill. Es indudable que el principialismo es una ayuda imprescindible para cualquier profesional del Trabajo Social. No obstante, los principios éticos se sitúan en la dimensión normativa, deontológica, de la ética profesional, cuya función es principalmente la de orientar la conducta interventiva del profesional. ¿Qué ocurre entonces cuando el profesional se enfrenta a una intervención concreta? ¿Es suficiente pensar sólo desde un punto de vista normativo o principialista? Es precisamente en la dimensión pragmática de la ética profesional donde surgen muchos problemas concretos y donde el trabajador o trabajadora social necesitará utilizar otras herramientas y otras perspectivas que puedan servirle de guía u orientación.

La ética de la alteridad (E. Lévinas) y la ética del cuidado (C. Gilligan) comparten que el uso exclusivo y racional de los principios puede dejar en un segundo plano la afectividad humana. El principialismo racionalista promueve que el sujeto moral se centre en cumplir con los principios, a la par que impulsa a tratar al Otro de modo generalizado. En cambio, las propuestas de Lévinas y de Gilligan nos llevan más allá de los principios, tomando éstos como base a partir de la cual entra en juego la afectividad humana que lleva al profesional a considerar al usuario en su concreta e irrepetible singularidad. Veamos más detalladamente ambas propuestas.

\section{La ética de la alteridad de E. Lévinas}

En la obra de Emmanuel Lévinas tuvo mucho que ver el horror del genocidio nazi que tuvo que padecer. Si antes del ascenso de Hitler al poder Lévinas ya intuía los derroteros a los que aquel incipiente fascismo abocaría al mundo, con posterioridad, aquella crítica se recrudecería, se volvería densa y compleja, para sintetizarse en su obra cumbre, Totalidad e Infinito. La ética propuesta por Lévinas es planteada en confrontación con las filosofías de Edmund Husserl y Martin Heidegger. La denuncia de Lévinas apunta a que el idealismo trascendental de Husserl acaba transformando al Otro en un alter ego (otro yo), mientras que en el realismo ontológico propuesto por Heidegger el Dasein («ser ahí») totaliza la singularidad del Otro, reduciéndola a la medida del ser. Lévinas no se cansará de criticar las consecuencias del totalitarismo al que arrastra la ontología. La ontología privilegia al sujeto a costa de la vida del Otro. Para la ontología el Otro es secundario, un alter ego del dominio 
apropiativo del sujeto. Un sujeto que se mantiene inalterable, perseverando en su identidad, mismificando cada adversidad, fagocitando cada contratiempo que la pueda poner en peligro.

El sujeto que se mantiene siempre el mismo -denominado por ello por Lévinas Mismo- reduce al Otro a sus categorías cognitivas, reduce la vida del Otro a sus ideas. La adecuación de lo Otro a lo Mismo se produce debido a la sincronización, a la presencia del sujeto ontológico sobre el Otro. La conciencia primera y prioritariamente ajusta la vida del Otro a la categoría mental del sujeto, considerándolo únicamente en su fenomenalidad aparente. El abordaje exclusivamente racional que promueve la ontología lleva al Mismo a considerar al Otro por su fachada fenoménica ${ }^{3}$.

La pregunta de Lévinas no se hace esperar: ¿pero es el Otro sólo la fachada que capta el Mismo? Y aunque lo fuera, ¿es este motivo suficiente para ejercer violencia sobre el Otro? Frente a una visión de la otra persona exclusivamente racional, Lévinas plantea aquella sensibilidad a la que somos despertados gracias al rostro del Otro. El rostro del Otro, aunque es habitualmente abordado por el Mismo como fenomenalidad, tiene la capacidad de conmover nuestra entraña profunda antes de que nuestra conciencia cognitiva pueda tomar parte en dicho proceso. El rostro nos afecta corporalmente -antes que cognitivamente- en diacronía, es decir, siempre antes de que nuestra voluntad pueda intervenir. El rostro no es reductible a categorías mentales del sujeto porque precede y excede al Mismo, conmoviéndolo preontológicamente. Esta conmoción preontológica que suscita el rostro en el sujeto es denominada por Lévinas sensibilidad. Una sensibilidad que significa incontrolable susceptibilidad frente al sufrimiento ajeno, es decir, no poder dejar de responder frente a su dolor, frente a su súplica exigente.

Desde el momento en el que el sujeto se sienta conmovido por el rostro, la sensibilidad a la que es despertado le exigirá mantenerse en adelante vigilante ante todo intento por su parte de categorizar completa y definitivamente al Otro como venía haciéndolo con anterioridad a dicha irrupción del rostro. A partir del momento en el que el rostro afecta corporalmente a un sujeto vulnerable y pasivo al que precede y excede, este sujeto, tras cada operación ontológica de cálculo, medida o comparación de la alteridad, tratará por todos los medios de devolverle su exclusiva singularidad volviéndole a mirar al rostro. Mirar al rostro suscita la respuesta prevoluntaria de responsabilidad del

3. Fenoménica en el sentido original del término «fenómeno», como aquello que aparece, que se nos muestra. Recordemos, en este sentido, la distinción kantiana entre «noúmeno» (las cosas en sí mismas) y «fenómeno» (cómo se nos muestran o aparecen las cosas). 
sujeto o ética, al auspicio de la cual se mantiene toda utilización del lenguaje de corte que es la ontología. Por ello, tener la ontología como primera filosofía lleva al sujeto a abordar al Otro reduciéndolo a sus categorías mentales, sin preocuparse de descategorizarlo, mientras que tener la ética como primera filosofía lleva al sujeto a abordarlo por la sensibilidad preoriginaria que le mantendrá en adelante vigilante ante toda tendencia altericida del lenguaje ontológico que utilice en toda operación de cálculo, medida y comparación.

La propuesta ética de Lévinas consiste en plantear la sensibilidad preoriginaria como modo de humanizar la ontologizada actividad del sujeto que, en la Europa occidental, tiende a ensalzar sobre todo la libertad, a cuyo amparo se encuentra la responsabilidad. Lévinas alude a que todo esto es consecuencia de que la filosofía solamente ha contemplado su aspecto griego, prescindiendo del hebreo, lo que ha llevado a que el sujeto aborde la realidad a través de tales operaciones ontológicas de cálculo, medida y comparación. El aspecto griego de la filosofía consiste en jerarquizar la realidad, en utilizar un lenguaje de corte para clasificarla, en presentificarla, en sincronizarla para mostrarla adecuada a unas categorías previamente establecidas, mientras que el aspecto hebreo es aquel que contempla la resistencia de la realidad a ser tematizada, habida cuenta de que la realidad se constituye de su sombra, jamás tematizable, que resulta irreductible porque precede y excede a toda posible tematización.

Para Levinas Occidente sólo considera el aspecto griego de la filosofía y por ello aborda al Otro considerando únicamente su fenomenalidad tematizable. Pero, ¿es el Otro un alter ego (otro yo) a la medida del Mismo? Occidente ha promovido que el Mismo reduzca al Otro a su fenomenalidad, tomando la parte (fachada reductible) por el todo (singularidad irreductible). De ahí la grandilocuencia que ha adquirido el conocimiento de la realidad: saber es poder desde el momento que se considera al Otro un alter ego de mi dominio apropiativo al que capto por el conocimiento que tengo de él. El Otro es lo que yo sé de él. Si sé de él lo puedo manejar, manipular, dominar. Por ello Lévinas considera que la ontología siempre se debe encontrar al auspicio de la ética, que toda operación ontológica, como el conocimiento, debe ser amparada por la sensibilidad preoriginaria que suscita en el sujeto la vigilancia por la que devolverá su singularidad al Otro tras cada categorización.

A modo de síntesis señalaremos que frente al idealismo trascendental de Husserl y la ontología de Heidegger, Lévinas propone la ética como primera filosofía. Que frente al sujeto trascendental husserliano cuya máxima aspiración es saber transformando al Otro por la intencionalidad noético-noemática en alter ego, y frente al Dasein («ser ahí») heideggeriano que acaba integrando 
al Otro a la medida del Ser, Lévinas plantea el Otro absolutamente Otro, como singularidad irreductible debido a que siempre precede y excede al sujeto que lo recibe. Frente al imperativo categoríco kantiano que establecía que la ética debía ser a priori (al margen de cualquier experiencia sensible) categórica (no condicionada por el exterior) y autónoma (aquélla que el sujeto se imponga racionalmente a sí mismo), Lévinas se cuestiona si la moral siempre ha de ser un conjunto de pautas de conducta, de principios abstractos que se aplican a priori a sujetos singulares y concretos.

Siguiendo con sus propios argumentos, dicho principialismo kantiano no hace sino totalizar la infinitud inconmensurable de la singularidad irreductible del Otro, es decir, reducir lo Otro a lo Mismo, el infinito a las categorías cognitivas totalizantes del sujeto que lo recibe. Para Lévinas, desde Parménides, pasando por Kant, Husserl y Heidegger, la ética ha permanecido al auspicio de la ontología, es decir, ha permanecido supeditada a la ley moral (Kant), al conocimiento (Husserl), al Ser (Heidegger).

De este modo, cuando la ontología es primera filosofía y la ética se encuentra a su amparo, se categoriza definitivamente la singularidad del Otro, puesto que no hay ética independiente de la ontología que pueda velar por descategorizarlo. Dicho de otro modo, debido a que la ética se encuentra subyugada por la ontología, no puede exigirle al sujeto nada que la ontología no ordene. Si fuera primera filosofía, la ética podría ordenar a la ontología la descategorización, pero, en ausencia de dicha ética, ¿quién velará por ello? La ontología como primera filosofía aboca a la categorización definitiva, sin que ninguna instancia independiente de ella nada pueda hacer para liberar a la singularidad ajena del catre de Procusto ${ }^{4}$ que sería, para Lévinas, el Ser.

De ahí la importancia de tener la ética como primera filosofía, estableciendo la sensibilidad, la responsabilidad y la vigilancia extrema como nociones clave de dicha propuesta: la sensibilidad es despertada por el rostro del Otro que, siempre a nuestro pesar, nos conmina a no poder dejar de responder. Dicha respuesta prevoluntaria de responsabilidad para con el Otro instaura una vigilancia extrema que consiste en velar por impedir que cuando categorizamos, no lo hagamos de forma definitiva. Dicha vigilancia extrema que se

4. En la mitología griega, Procusto era el apodo de un posadero llamado Eleusis. Le llamaban Procusto (que significa «el estirador») porque obligaba a acostarse en una cama de hierro a los huéspedes, serrando o estirando los pies y piernas de quienes no se ajustaban al tamaño exacto de la cama. El significado de este mito es que hay personas que pretenden siempre acomodar la realidad a su particular perspectiva de las cosas. La actitud contraria a la de Procusto sería la de no tratar de interpretar a todos los demás con nuestros propios moldes, la de observar, escuchar, ser receptivos y abiertos a cada persona. 
encuentra investida por la sensibilidad preoriginaria exige al sujeto que, tras cada categorización del Otro, volvamos a mirarle al rostro para devolverle su singularidad. En definitiva, la vigilancia extrema es la que obliga al sujeto a la atención crítica permanente en cada operación ontológica de cálculo, medida y comparación. Los principios éticos, como operaciones ontológicas, son investidas en adelante por la sensibilidad y la vigilancia extrema. Se garantiza así la irreductibilidad de la singularidad del Otro.

Tener la ética como primera filosofía hace que la aplicación de los principios éticos se encuentre investida por aquella sensibilidad preoriginaria que velará en todo momento por impedir la categorización definitiva del Otro, siendo que -tras cada categorización- el sujeto volverá a mirar al Otro al rostro, en cuyo caso se producirá la descategorización y el mantenimiento de la irreductibilidad de la singularidad del Otro. En definitiva, el olvido de la sensibilidad promueve que el sujeto trate a su prójimo como Otro abstracto, general, como elemento que forma parte de la totalidad de un sistema impersonal y anónimo. Mientras que aplicar los principios éticos investidos por la sensibilidad, garantiza la irreductibilidad de la singularidad del Otro concreto.

Como sucede con la ética del cuidado de Carol Gilligan que expondremos a continuación, la importancia de la aportación levinasiana radica en la incorporación de la noción de sensibilidad. Del mismo modo que Schopenhauer, Nietzsche, Merleau-Ponty o Husserl, Lévinas es otro de los filósofos que devuelve al cuerpo y su sensibilidad la dignidad perdida a lo largo de los siglos, dándole máxima importancia y prioridad en su propuesta ética. Gracias a Platón y a Descartes, el cuerpo se encuentra supeditado a una instancia superior: al alma en Platón, al cógito en Descartes. Merleau-Ponty será el que considere el cuerpo aquello que se tiene a la par que aquello que se es: se tiene y se es cuerpo. Para Lévinas la corporalidad se ve afectada en diacronía. Es aquella vulnerabilidad pasiva por la que el sujeto se encuentra abocado a responder. Esta vulnerabilidad pasiva de la corporeidad afectada es denominada por Lévinas deseo metafísico y preontológico: deseo irrefrenable de servir al Otro. Lévinas fue más allá de lo que lo hiciera su maestro Husserl con respecto a la corporalidad. Si bien es cierto que Husserl consideraba al cuerpo -y no sólo a la conciencia- como algo importante para la percepción ${ }^{5}$, Lévinas otorga más importancia a la corporalidad que a la conciencia cognitiva en su propuesta ética.

5. Cfr. Husserl, 1962: 88-89. 


\section{La ética del cuidado de Carol Gilligan.}

Del mismo modo que le sucediera a Lévinas, Gilligan elabora su propuesta ética en confrontación con quien fuera su profesor en Harvard, Lawrence Kohlberg. La propuesta ética de Kohlberg surge fruto de un estudio realizado con varones a los que se les plantea una serie de dilemas hipotéticos. La conclusión a la que llega es que las mujeres tienen un desarrollo moral inferior que los hombres. Por su parte, Gilligan realiza el estudio con mujeres a las que plantea dilemas reales. Su conclusión: las mujeres tienen diferente desarrollo moral que los hombres debido a su diferente socialización. Resulta admirable que la ética del cuidado que Gilligan propone no sea contra la ética de Kohlberg, sino como complemento de ésta. De hecho, es considerada como una ampliación de la propuesta de su profesor.

Mientras que para Kohlberg el Otro se concibe generalizado -como haciendo abstracción de lo concreto a lo general-, para Gilligan el Otro es concebido como singularidad concreta en su contexto circunstancial, pasando de lo general a lo concreto. Para Kohlberg el sujeto moral tiende a ponerse en lugar del Otro, que consiste en pensar o imaginar lo que el sujeto haría si estuviera en una piel y una circunstancia en las que nunca estará y que, a la hora de hacer dicha abstracción, desconoce por completo. Por su parte, Gilligan propone la importancia de las relaciones personales en las que, lejos de ponerse intelectual o imaginariamente en el lugar del Otro, aboga por cuidarlo. El Otro no es un alter ego producto de mi pensamiento o de mi imaginación y por ello alguien abstracto. El Otro para Gilligan es alguien vulnerable y por ello necesitado de nuestro cuidado.

Fiel al principialismo kantiano, Kohlberg considera fundamental la igualdad y la imparcialidad en el trato al Otro generalizado. La razón de ser de esta concepción radica en que los principios han de ser aplicados sin que para ello se tenga que conocer la situación concreta: principios idénticos exigen ser aplicados en circunstancias muy diferentes. ¿Se puede tratar al diferente del mismo modo que a todos los demás? Anticipamos que Mary Richmond, una de las fundadoras del Trabajo Social, afirmó: hay que tratar al diferente siempre de forma diferente. En esta línea, para Gilligan la propuesta igualitarista de Kohlberg no sólo le parece que acaba violentando al Otro, sino que lo oprime por ser una imposición autoritarista. ¿Cómo podría ser tratado alguien que va en silla de ruedas del mismo modo que otra persona que no tiene dificultades de movilidad? Por ello, dos nociones en las que insiste Gilligan son la consideración de la diversidad, así como la del contexto situacional del Otro concreto a través de las relaciones personales. Como veremos, esto es 
algo crucial en el Trabajo Social, puesto que nuestra herramienta fundamental de trabajo es la relación interpersonal con el usuario.

La relación personal nos lleva a conocer al Otro concreto, frente a la consideración del Otro generalizado que es fruto de nuestro pensamiento intelectual y abstracto. La relación personal nos acerca a la realidad concreta del Otro concreto. La neutralidad de la igualdad y la imparcialidad propuestas por Kohlberg nos distancian de un sujeto al que sustituimos por lo que nosotros creemos que es el Otro. De hecho, como anticipábamos, ponernos en el lugar del Otro es una simple proyección de lo que nosotros mismos haríamos en un cuerpo y en un contexto que no conocemos. Cuando, en tales circunstancias aludimos al Otro, nos estamos refiriendo a lo que nosotros imaginamos que haríamos en su lugar. Pero el Otro es de carne y hueso. Como dijera Lévinas, «el sujeto es de carne y sangre (...) entrañas en una piel» (Levinas 2003: 136) ${ }^{6}$. El Otro es Otro diferente que nosotros y no una idea nuestra.

Para Gilligan, también el Otro es Otro diferente a nosotros y por ello debemos tratar de conocerlo lo máximo y lo más profundamente posible, tomándonos el tiempo necesario para ello. Mientras que para Kohlberg el sujeto moral sería un sujeto racional con derechos y deberes que cumplir, para esta autora, el sujeto ético es un sujeto que se preocupa del Otro porque lo concibe en su vulnerabilidad, necesitado de cuidado. Kohlberg se centra en lo que debe hacer el sujeto moral, mientras que Gilligan se centra en cuidar del que lo necesita. Ésta es una de las grandes diferencias que Lévinas explicaría del siguiente modo: la ética de la justicia de Kohlberg, centrada en el Mismo, se encuentra ontologizada, mientras que la ética del cuidado de Gilligan, centrada en el Otro, no lo estaría.

Por tanto, el universalismo practicado por Kohlberg sería un universalismo sustitucional (aquel proceso de universalización por el que el Otro pierde su singularidad, su unicidad exclusiva por integrarse en un todo ordenado, en la totalidad de un sistema), mientras que el practicado por Gilligan sería un universalismo interactivo (aquel proceso de universalización en el que la singularidad del Otro es respetada, dado que tras la categorización abstracta se vuelve a cuidar del Otro concreto). En esta misma línea, en la ética de la justicia de Kohlberg lo que prepondera es la abstracción de lo concreto a lo general, las operaciones ontológicas abstractas y generales, mientras que en la ética del cuidado de Gilligan lo que predomina es el paso de lo abstracto a lo concreto, es decir, lo local, lo circunstancial, el contexto situacional. En definitiva, mientras que la propuesta de Kohlberg tiende a intelectualizar al Otro,

6. También Cfr. Lévinas, 2003: 132, 137. 
Gilligan propone el cara a cara de las relaciones personales en las que surge de manera natural la necesidad de cuidar del Otro concreto.

Uno de los aspectos que tienen en común la ética de la alteridad de Lévinas y la del cuidado de Gilligan es la importancia que otorgan a la afectividad humana como determinante en sus propuestas. Si bien es cierto que Kohlberg no niega lo afectivo, lo cierto es que no le confiere la importancia necesaria. Su ética promueve un contexto intelectualizante que principalmente vela por el cumplimiento estricto de una serie de principios y reglas abstractas. En cambio, tanto en la ética de Lévinas como en la de Gilligan, la consideración de lo emocional es lo que las diferencia. Para Lévinas la sensibilidad es la que sustenta la responsabilidad y la consiguiente vigilancia extrema, mientras que para Gilligan la afectividad es inherente a una relación personal en la que ésta surge de forma espontánea como necesidad de cuidar del Otro concreto. De hecho, y probablemente de la misma manera que para Lévinas dicha sensibilidad es la que nos impulsa a la excelencia de todas las operaciones ontológicas, para Gilligan el afecto que surge en la relación con el Otro concreto es lo que le lleva al sujeto ético a saber el máximo posible y en profundidad del Otro que lo llevará a cuidarlo siempre mejor.

\section{La repercusión de las éticas de la alteridad y del cuidado en el Trabajo Social}

Todo lo anteriormente expuesto nos lleva a considerar que ambas éticas son planteamientos que tienen una gran aplicación al Trabajo Social, puesto que pueden servir como complemento al principialismo ético universal y pueden aportar otra perspectiva en las intervenciones con los usuarios: la de la sensibilidad, la implicación afectiva, el cuidado, concreción y contextualización del Otro.

La sensibilidad que propone Lévinas lleva al sujeto ético, antes que ninguna otra cosa, a responder prevoluntariamente ante el sufrimiento ajeno, garantizando de ese modo que el Otro no quede desatendido, así como a mantenerse vigilante impidiendo categorizar definitivamente al Otro en el ejercicio de sus operaciones ontológicas. Por su parte, Gilligan apuesta por aquella implicación afectiva que surge en el cara a cara de la relación interpersonal de cuidado, que es la que le llevará a conocer más en profundidad a ese Otro que no conoce pero que desea hacerlo para poderle ayudar, para poder dar salida al deseo de cuidarlo de la mejor manera posible.

Por otra parte, como ya lo anticipábamos al comienzo de este artículo, en el Trabajo Social se ha desarrollado mucho más la dimensión normativa que la dimensión pragmática de la ética profesional. En este sentido, la ética 
de Lévinas y de Gilligan podrían ser perspectivas que nos acercasen más al usuario concreto. De hecho, para ambas propuestas, el principialismo racional universal supondría tratar al usuario en relación a principios abstractos establecidos a priori. Si desde la perspectiva levinasiana consideramos que tales principios éticos son operaciones ontológicas, la investidura de éstos por la sensibilidad preoriginaria traería consigo una mayor humanización de los mismos, pues serían tenidos en consideración en el contexto de cada caso concreto, siempre a posteriori de la irrupción del rostro.

Del mismo modo, Gilligan deja claro que su ética del cuidado es un complemento que amplía la ética de la justicia de Kohlberg. Ambas propuestas pueden considerarse complementos que amplían el enfoque principialista predominante en Trabajo Social, intentando tomar como base la noción de proximidad. Frente a la distancia que, para ambos autores, produce el sólo principialismo, el imperativo de acercamiento que trasladan sus propuestas puede resultar muy beneficioso para la intervención social en el Trabajo Social. Si para ambos la ética se produce en relación social y ésta resulta ser una de las herramientas principales de trabajo para dicha disciplina, creemos que la incorporación de ambas al Trabajo Social podría llegar a enriquecer más su intervención social.

Ambas éticas apuestan principalmente por la consideración en la relación cara a cara de un elemento como el emocional que no ha sido tenido en cuenta con la pertinencia y relevancia que se debiera. Es innegable y evidente la trascendencia que ha tenido y continúa teniendo el principialismo ético racional en nuestra profesión. Pero quizá no baste sólo con aplicar los principios éticos -al menos de una forma exclusivamente racional y abstracta- en las intervenciones profesionales. Si sólo consideramos a los usuarios como otro generalizado abstracto y universal, olvidamos la concreción del Otro en cada sujeto concreto que despierta, casi inevitablemente, nuestra sensibilidad preracional y nuestras emociones. Si nos centramos en el Otro concreto, es entonces cuando surge, en el cara a cara, la sensibilidad (Lévinas) y la implicación afectiva (Gilligan) para con el Otro. Tener en cuenta la emoción como componente importante de sus propuestas nos lleva a humanizar más la intervención social.

\section{Conclusión}

La ética de la alteridad de Lévinas y la ética del cuidado de Gilligan pueden resultar un complemento humanizante para las intervenciones profesionales en Trabajo Social. Tener la ética como primera filosofía o apostar por el cuidado del Otro concreto no son sino propuestas que humanizarían más las 
intervenciones con los usuarios. Desde la ética de Lévinas, por la sensibilidad preoriginaria que nos despierta el rostro del usuario no sólo se nos conmina a responderlo prevoluntariamente, sino que instaura en nosotros una vigilancia extrema tal que nos exige velar por que detrás de todas las operaciones ontológicas de cálculo, medida y comparación (que en Trabajo Social serían las operaciones de evaluación, diagnóstico, catalogación, comparación, clasificación, aplicación de los principios éticos al caso concreto, etc.), volvamos a mirar nuevamente al rostro del usuario, devolviéndole así su singularidad y protegiendo su decisión autónoma. Los principios éticos, como operación ontológica, quedarían investidos por la sensibilidad preoriginaria. Teniendo la ética como primera filosofía, los principios éticos universales se humanizan al ser considerados en el contexto del caso concreto gracias a la sensibilidad.

La ética de Gilligan también supone un plus de humanización de la intervención en Trabajo Social, pues la implicación afectiva con el usuario surge en relación interpersonal con él. Sólo en el cara a cara con el usuario surge el deseo de cuidar al Otro concreto, al que deseamos conocer en profundidad para poder cuidar de él de la mejor manera posible. En comparación con la propuesta de Lévinas, queremos destacar la sencillez y simplicidad de la propuesta de Gilligan, que es capaz de derivar de forma natural la necesidad de cuidado del sujeto ético de la vulnerabilidad que reconoce en el usuario y que le implica emocionalmente en la relación interpersonal que mantiene cara a cara con él. El reconocimiento de la vulnerabilidad, que surge como implicación afectiva en relación interpersonal con el usuario, es la que lleva al profesional a cuidar de él.

En definitiva, creemos que las éticas de Lévinas y de Gilligan suponen una importante contribución para la ética del Trabajo Social. La de Lévinas, por impedir categorizar definitivamente la singularidad irreductible del usuario, y la de Gilligan, por descender al cara a cara afectivo en nuestras relaciones con los usuarios.

\section{Bibliografía}

Alonso, R., Fombuena, J. (2006). La ética de la justicia y la ética de los cuidados. Portularia, 6 (1), 95-107.

Ballestero, A. (2009). Dilemas éticos en Trabajo Social: El modelo de la Ley Social. Portularia, 9 (2), 123-131. Recuperado el 10 de Noviembre de 2010 de http://redalyc.uaemex.mx/pdf/1610/161013165008.pdf.

Comins, I. (2003). Del Miedo a la Diversidad a la Ética del Cuidado: Una Perspectiva de Género. Convergencia, 33, 97-122. Recuperado el 10 de Noviembre de $2010 \mathrm{de}$ http://convergencia.uaemex.mx/rev33/33pdf/4_irene_comins.pdf. 
Gilligan, C. (1982). In a Different Voice: Psychological Theory and Women's Development. Cambridge, Mass: Harvard University Press (Traducción castellana: Moral y Teoría: Psicología del desarrollo femenino. México: Fondo de Cultura Económica. 1994).

Guzman, F, Toboso, M., \& Romañach, J. (2010). Fundamentos éticos para la promoción de la autonomía: hacia una ética de la Interdependencia. Alternativas. Cuadernos de Trabajo Social, 17, 45-61. Recuperado el 10 de Noviembre de 2010 de http://digital.csic.es/bitstream/10261/26666/1/Guzm\%c3\%aln_ Toboso_Roma\%c3\%blach_Alternativas_17_2010.pdf.

HUSSERL, M. (1962). Ideas relativas a una fenomenología pura y una filosofía fenomenológica. México: FCE.

Koggel, C. \& Orme, J. (2010). Care Ethics: New Theories and Applications. Ethics and Social Welfare, 4(2), 109-114. Recuperado el 10 de Noviembre de 2010 de http://pdfserve.informaworld.com/435603_923316937.pdf.

LÉVINAS E. (1991). La ética. En J. CASADO \& P. AGUDÍEZ (Comps). El sujeto europeo (pp. 3-15). Madrid: Pablo Iglesias.

LÉVINAS, E. (1993). Dios, la muerte y el tiempo. Madrid: Cátedra.

LÉVINAS, E. (2000). Ética e infinito. Madrid. A. Machado.

LÉVINAS, E. (2001). La realidad y su sombra: libertad y mandato, trascendencia y altura. Madrid: Trotta.

LÉvinAS, E. (2003). De otro modo que ser o más allá de la esencia. Salamanca: Sígueme.

LÉVINAS, E. (2006a). Humanismo del otro hombre. Madrid: Siglo XXI.

LÉVINAS, E. (2006b). Totalidad e Infinito: ensayo sobre la exterioridad. Salamanca: Sígueme.

LÉVINAS, E., \& KEARNEY, R. (1998). Ética del Infinito. En R. KEARNEY. La paradoja europea (pp. 197-218). Barcelona: Tusquets.

LÉVINAS, E., \& POIRIÉ, F. (2009). Conversaciones. En F. POIRIÉ \& E. LÉVINAS. Ensayo y Conversaciones (pp. 49-116). Madrid: Arena.

SAlcedo, D. (2001). Autonomía y bienestar. La ética del Trabajo Social. Granada: Comares.

SALCEDO, D. (2010). Los fundamentos normativos de las profesiones y los deberes de los trabajadores sociales. Trabajo Social Global, 1(1), 10-38.

SANTACRUZ, M ${ }^{\mathrm{a} C}$. (2006). Ética del cuidado. Universidad del Cauca. Recuperado el 10 de Noviembre de 2010 de http://www.facultadsalud.unicauca.edu.co/ fcs/2006/junio/ETICA\%20Y\%20CUIDADO.pdf.

Úriz PemÁn, Maj. (2009, Mayo). El 'buen gusto ético': distintos sabores para una misma ética profesional. Recuperado el 24 de Abril de 2010 de la Web del Consejo General de Colegios Oficiales de Diplomados en Trabajo Social y Asistentes Sociales: http://www.cgtrabajosocial.es/alicante/documentos/ congreso/31_M_Jesus_Uriz.pdf 